\title{
Zanamivir Diminishes Lung Damage in Influenza A Virus-infected Mice by Inhibiting Nitric Oxide Production
}

\author{
BIRUTĖ ZABLOCKIENE ${ }^{1}$, TOMAS KAČERGIUS ${ }^{2,3}$, ARVYDAS AMBROZAITIS ${ }^{1}$, \\ EDVARDAS ŽURAUSKAS ${ }^{4}$, MAKSIM BRATCHIKOV ${ }^{2}$, LAIMUTE் JURGAUSKIENE ${ }^{5}$, \\ ROLANDAS ZABLOCKIS ${ }^{1}$ and STEFAN GRAVENSTEIN ${ }^{3,6,7}$ \\ ${ }^{I}$ Clinic of Infectious and Chest Diseases, Dermatovenerology and Allergology, and \\ ${ }^{5}$ Clinic of Cardiovascular Diseases, Institute of Clinical Medicine, and ${ }^{2}$ Department of Physiology, \\ Biochemistry, Microbiology and Laboratory Medicine, and ${ }^{4}$ Department of Pathology, \\ Forensic Medicine and Pharmacology, Institute of Biomedical Sciences, \\ Faculty of Medicine, Vilnius University, Vilnius, Lithuania; \\ ${ }^{3}$ Department of Internal Medicine, Glennan Center for Geriatrics and Gerontology, \\ Eastern Virginia Medical School, Norfolk, VA, U.S.A.; \\ ${ }^{6}$ Department of Medicine, Warren Alpert Medical School, and \\ ${ }^{7}$ Department of Health Services Policy and Practice, School of Public Health, \\ Brown University, and Providence Veterans Administration Hospital, Providence, RI, U.S.A.
}

\begin{abstract}
Background/Aim: Severe pulmonary influenza A virus (IAV) infection causes lung inflammation and expression of inducible nitric oxide synthase (iNOS), leading to overproduction of nitric oxide (NO). We studied whether zanamivir reduces pulmonary inflammation through inhibition of NO production in mice. Materials and Methods: We treated IAV-infected mice daily with intranasal zanamivir. Controls were infected and either placebo-treated or untreated, or not infected and placebo-treated. Mice were weighed daily. After euthanasia on day 3, lungs were excised and bronchoalveolar lavage was performed and fluid nitrite concentration was determined. Lungs were analyzed microscopically. iNOS and IAV RNA levels in lungs were assessed using quantitative reverse transcriptionpolymerase chain reaction (RT-qPCR). Results: Mice undergoing zanamivir treatment had less weight loss, viral replication, and lung damage, as well as significant reductions of local NO and iNOS mRNA synthesis $(p<0.05)$. Conclusion: Zanamivir is associated with an anti-inflammatory effect mediated through inhibition of NO production in IAV-infected mice.
\end{abstract}

This article is freely accessible online.

Correspondence to: Birutė Zablockienè, Clinic of Infectious and Chest Diseases, Dermatovenerology and Allergology, Institute of Clinical Medicine, Faculty of Medicine, Vilnius University, Birutes str. 1, LT-08117, Vilnius, Lithuania. Tel: +370 61143253, e-mail: Birute.Zablockiene@santa.lt

Key Words: Influenza virus, lung pathology, nitric oxide, zanamivir.
Influenza A virus infection (IAV) remains a persistent threat to human health worldwide. Despite the availability of vaccinations, seasonal influenza epidemics result in 3 to 5 million cases of severe illness, and about 290,000 to 650,000 deaths annually (1). Severe disease is associated with initial primary viral pneumonia causing excessive lung inflammation and eventually acute respiratory distress syndrome and bacterial pneumonia (2). IAV infection induces production of pro-inflammatory cytokines and recruitment of inflammatory cells, such as neutrophils and macrophages, to the infected lungs (2). Cytokines such as interferon-gamma (IFN- $\gamma$ ) and tumor necrosis factor-alpha (TNF- $\alpha$ ) stimulate inducible nitric oxide synthase (iNOS), leading to high-output synthesis of a free radical, namely nitric oxide (NO), which occurs predominantly in macrophages $(2,3)$. In turn, overproduction of NO and subsequent formation of reactive nitrogen species (e.g. peroxynitrite) can injure lung tissue $(4,5)$. Thus, it is important to design new therapeutic approaches directed against iNOS expression.

Zanamivir (Relenza ${ }^{\mathrm{TM}}$ ) is an inhaled viral neuraminidase inhibitor which demonstrated effectiveness for reducing pulmonary virus titers, lung consolidation, and morbidity and mortality scores in IAV-infected mice, as well as for alleviating clinical symptoms of influenza-infected humans (6-8). Importantly, it was also found to suppress production of NO in IAV-infected and IFN- $\gamma$-activated RAW 264.7 macrophages in vitro (9). Therefore, in this study, for the first time, we tested the capacity of zanamivir to reduce pulmonary inflammation through the inhibition of NO synthesis under in vivo conditions, i.e. in IAV-infected mice. 


\section{Materials and Methods}

Animals. Female BALB/c mice (6-12 weeks of age) weighing 18-20 g were obtained from Charles River Laboratories, Inc., Wilmington, MA, USA. The mice were housed within microisolator cages in an isolation room, and all experimental procedures were performed in the biosafety cabinet using biosafety level 2 containment. The animals were anesthetized by intraperitoneal injection of a mixture of ketamine (50 $\mathrm{mg} / \mathrm{kg}$ ) and xylazine $(50 \mathrm{mg} / \mathrm{kg})$ before every procedure: inoculation with virus, treatment with compounds and euthanasia. All protocols involving experiments with mice were approved by the Institutional Animal Care and Use Committee, Eastern Virginia Medical School, Norfolk, VA, USA (permit no. 07-003). The animal experiments were carried out within the provisions of the Animal Welfare Act (Public Law 99-198), the National Research Council, the Public Health Service Policy on Humane Care and Use of Laboratory Animals, the "Guide for the Care and Use of Laboratory Animals (1996)", the Health Research Extension Act of 1985 Public Law 99-158 (11/20/86), and United States Department of Agriculture regulations.

Influenza virus infectivity. The mouse-adapted influenza A/Puerto Rico/8/34 (A/PR/8/34, H1N1) virus was received from Dr. Bradley S. Bender (College of Medicine, University of Florida, Gainesville, FL, USA), and its infectivity titer of $50 \%$ tissue culture infectious dose (TCID50) of 108.4/ml was determined in Madin-Darby canine kidney cells (ATCC CCL-34), as previously described (9). Afterwards, mice were sequentially challenged with a single $20 \mu \mathrm{l}$ dose at a specific dilution of serial dilutions of the virus stock in suspension (10 $\mu 1 /$ nostril). Mice were then followed-up for 3 days in order to determine the virus infectivity titer that produced clinical infection without causing death (sublethal dose). Using body weight loss to determine clinical infection, and survival rate of mice, a virus infectivity titer of $107.6 \mathrm{TCID} 50 / \mathrm{ml}$ was determined as the optimal sublethal infectious concentration and selected for further experiments.

Compounds. Zanamivir (5-(acetylamino)-4-[(aminoiminomethyl)amino]-2,6-anhydro-3,4,5-trideoxy-D-glycero-D-galacto-non-2-enonic acid) was purchased from GlaxoSmithKline (Research Triangle Park, NC, USA) as the drug Relenza ${ }^{\mathrm{TM}}$ consisting of a powder mixture of $5 \mathrm{mg}$ of zanamivir and $20 \mathrm{mg}$ of lactose per blister. The dosage of zanamivir $(2 \mathrm{mg} / \mathrm{kg})$ for the mice was based on its therapeutic dose range $(0.3-12.5 \mathrm{mg} / \mathrm{kg}$ ) provided in the literature $(6,7)$. Lactose (as placebo) was obtained from Sigma-Aldrich (St. Louis, MO, USA). The dose of lactose (placebo) was $8 \mathrm{mg} / \mathrm{kg}$. The compounds were

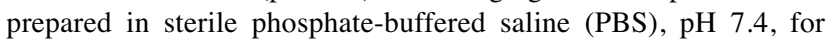
intranasal administration of $20 \mu \mathrm{l}(10 \mu \mathrm{l} /$ nostril $)$ to the mice.

Experimental design. The anesthetized mice were inoculated intranasally with $20 \mu \mathrm{l}$ of influenza A/PR/8/34 virus suspension with infectivity titer of $10^{7.6} \mathrm{TCID}_{50} / \mathrm{ml}$. At $3 \mathrm{~h}$ post-infection (p.i.), mice were initiated on a daily treatment with $2 \mathrm{mg} / \mathrm{kg}$ of zanamivir $(\mathrm{n}=4)$ or $8 \mathrm{mg} / \mathrm{kg}$ of lactose (Sigma-Aldrich) as a placebo $(\mathrm{n}=3)$. These doses of zanamivir and lactose were administered intranasally in $20 \mu \mathrm{l}(10$ $\mu 1 /$ nostril) of sterile PBS. Infected mice without treatment $(n=3)$ or receiving lactose as were uninfected mice treated with lactose $(n=3)$ under the same dosage and regimen served as controls. The compounds were administered in a single-dose regimen to minimize animal distress caused by treatment procedures. Animals were monitored daily for morbidity, as measured by body weight loss. After 3 days, all of the mice were euthanized by axillary bleeding (terminal exsanguination), and then lungs were excised and lavaged twice with $1 \mathrm{ml}$ of PBS via the cannulated trachea. The collected bronchoalveolar lavage (BAL) fluids were centrifuged at $735 \times g$ for $5 \mathrm{~min}$, and the supernatants were subjected to the determination of nitrite level. Immediately after the BAL procedure, the left lung was placed in $4 \%$ paraformaldehyde (Thermo Fisher Scientific, Waltham, MA, USA) at $4{ }^{\circ} \mathrm{C}$ until processing for histological examination. The right lung was immersed in TRIzol ${ }^{\mathrm{TM}}$ reagent (Thermo Fisher Scientific) and homogenized on dry ice using a Tissue Tearor (Dremel, Racine, WI, USA). The lung tissue homogenates were centrifuged at $12,000 \times g$ for $5 \mathrm{~min}$ at $4^{\circ} \mathrm{C}$, and the supernatants were frozen at $-80^{\circ} \mathrm{C}$. Afterwards, they were used for isolation of RNA followed by determination of the levels of viral RNA, iNOS and $\beta$-actin mRNA applying a multiplex one-step quantitative reverse transcription polymerase chain reaction (RT-qPCR) with fluorescent hydrolysis probes.

Nitrite determination. The level of nitrite $\left(\mathrm{NO}_{2}^{-}\right)$, an indicator of NO synthesis in biological systems, was determined in BAL fluids using a modified Griess reagent (Sigma-Aldrich) as directed by the manufacturer. A microplate-reader spectrophotometer PowerWave X 340 (BioTek Instruments, Inc., Winooski, VT, USA) was employed to measure the optical density (OD) of BAL fluid samples at a wavelength of $540 \mathrm{~nm}$. Nitrite concentrations were calculated using KC4 software, version 2.5 (BioTek Instruments, Inc.), by comparison of the OD of the samples to a standard curve of sodium nitrite. The lower limit of nitrite detection was $0.12 \mu \mathrm{mol} / \mathrm{ml}$.

Histological examination. The harvested lung tissues were embedded in paraffin, cut into $5 \mu \mathrm{m}$ sections and stained with hematoxylin and eosin (H\&E). Coded lung samples were examined using light microscopy by a pathologist in the blinded approach. The following indicative parameters of pulmonary histopathology were evaluated: perivascular lymphocytic, peribronchial lymphocytic, focal and diffuse leucocytic infiltrations; alveolar macrophages; damage to alveolar/bronchial epithelium; hyperemia; capillary thromboses; bronchiolitis; pleuritis; alveolar hemorrhage, collapse, fibrosis, edema and hyaline membranes. Each of these parameters was subjectively scored on a scale from 0 (absence of pathological changes) to 3 (maximum extent of pathological changes), and the sum of scores comprised the total histological score for each mouse.

RNA isolation and multiplex one-step RT-qPCR. Total RNA was isolated from lung homogenates using TRIzol ${ }^{\mathrm{TM}}$ reagent according to the instructions provided by Thermo Fisher Scientific. The levels of viral RNA [encoding the IAV polymerase acidic (PA) protein], iNOS and $\beta$-actin mRNA were determined by multiplex one-step RT-qPCR using SensiFAST ${ }^{\mathrm{Tm}}$ Probe No-ROX One-Step Kit (Bioline Reagents Ltd, London, UK). Primers and hydrolysis probes, comprised of the nucleotide sequences shown in Table I, were synthesized by Biolegio B.V., Nijmegen, the Netherlands. The primer and probe sequences were designed using Vector NTI Advance $^{\mathrm{TM}}$ program (Thermo Fisher Scientific) for sequence alignment and FastPCR online (http://primerdigital.com/ tools/pcr.html) Java applet for primer tests. All multiplex one-step reactions were carried out in a total volume of $15 \mu \mathrm{l}$ using $1 \mu \mathrm{l}$ of the isolated RNA sample and primers at a concentration of $200 \mathrm{nM}$ each, and probes at a concentration of $100 \mathrm{nM}$ each. The multiplex one-step RT-qPCR assays were performed employing a real-time thermocycler Rotor-Gene Q 5plex model with software version 1.7 (Qiagen $\mathrm{GmbH}$, Hilden, Germany) under the following conditions: 
Table I. Primer and probe sequences used in the multiplex one-step quantitative reverse transcription polymerase chain reaction.

\begin{tabular}{|c|c|c|c|}
\hline Gene & Primer/probe & Sequence & Amplicon size (bp) \\
\hline \multirow[t]{3}{*}{ IAV PA } & Forward primer & 5'-TCTCAGCGGTCCAAATTCCTGC-3' & 146 \\
\hline & Reverse primer & 5'-GGTTTAACAACATTGGGTTCCTTCCAT-3' & \\
\hline & Probe & 5'-HEX-TGAGGACCCAAGTCATGAAGGAGAGGGA-BHQ1-3' & \\
\hline \multirow[t]{3}{*}{ iNOS } & Forward primer & 5'-CTTGTGCTGTTCTCAGCCCAACAATA-3' & 95 \\
\hline & Reverse primer & 5'-TGGAACATTCTGTGCTGTCCCAG-3' & \\
\hline & Probe & 5'-FAM-TGGCTCCCCGCAGCTCCTC-BHQ1-3' & \\
\hline \multirow[t]{3}{*}{$\beta$-Actin } & Forward primer & 5'-GCACMATGAAGATCAAGATCATTGCTCC-3' & 118 \\
\hline & Reverse primer & 5'-TCRTACTCCTGCTTGCTGATCCAC-3' & \\
\hline & Probe & 5'-ROX-TCCTGGCCTCRCTGTCCACCTTCC-BHQ2-3' & \\
\hline
\end{tabular}

IAV PA: Influenza A virus polymerase acidic protein; iNOS: inducible nitric oxide synthase.

Table II. Body weights of treated and control groups for the 3 days following influenza A virus infection. The body weights are expressed as a percentage of their initial weight at day 0 before virus inoculation. Data are shown as mean \pm SEM.

\begin{tabular}{lccccc}
\hline GroupTreatment & \multicolumn{3}{c}{ Body weight (\%) } \\
\cline { 3 - 6 } & & Day 0 & Day 1 & Day 2 & Day 3 \\
\hline $\mathrm{A}, \mathrm{n}=3$ & Uninfected + placebo & 100 & $94.24 \pm 0.67$ & $91.41 \pm 1.83$ & $88.63 \pm 4.40$ \\
$\mathrm{~B}, \mathrm{n}=3$ & Infected & 100 & $95.13 \pm 0.92$ & $87.10 \pm 0.50$ & $82.27 \pm 1.47$ \\
$\mathrm{C}, \mathrm{n}=3$ & Infected + placebo & 100 & $94.99 \pm 0.41$ & $88.93 \pm 0.59$ & $83.57 \pm 0.90$ \\
$\mathrm{D}, \mathrm{n}=4$ & Infected + zanamivir & 100 & $95.32 \pm 0.79$ & $93.33 \pm 1.55^{* \#}$ & $91.23 \pm 2.07$ *\# \\
\hline
\end{tabular}

Significantly different at $p<0.05 v s$. *group B, and \#group C.

first strand cDNA was synthesized at $48^{\circ} \mathrm{C}$ for $10 \mathrm{~min}$ and then denature at $95^{\circ} \mathrm{C}$ for $2 \mathrm{~min}$; followed by 50 cycles of denaturation at $95^{\circ} \mathrm{C}$ for $10 \mathrm{~s}$ and annealing/extension at $60^{\circ} \mathrm{C}$ for $1 \mathrm{~min}$. The $2^{-\triangle \Delta C T}$ algorithm was applied for calculation of relative levels of viral PA RNA and iNOS mRNA (10). The expression of $\beta$-actin mRNA was used for normalization of the relative viral PA RNA and iNOS mRNA levels between different samples.

Statistical analysis. Differences between the experimental groups were evaluated applying one-way analysis of variance (ANOVA) followed by a post hoc least significant difference (LSD) test for multiple comparisons. Data are presented as means \pm standard error of the mean (SEM). A $p$-value of less than 0.05 was considered to indicate a statistically significant difference. Data were analyzed using the Statistical Package for Social Science software version 21.0 for Windows (IBM Corp., Armonk, NY, USA).

\section{Results}

Body weight changes. The daily body weights of mice for the 3-day experiment are shown in Table II. All mice lost weight. On days 2 and 3 , the uninfected mice receiving placebo lost approximately $9 \%$ and $11 \%$ of their initial body mass. In contrast, the infected as well as infected placebotreated mice had a weight loss of $13 \%$ and $11 \%$ on day 2 as well as $18 \%$ and $16 \%$ on day 3 from their original body

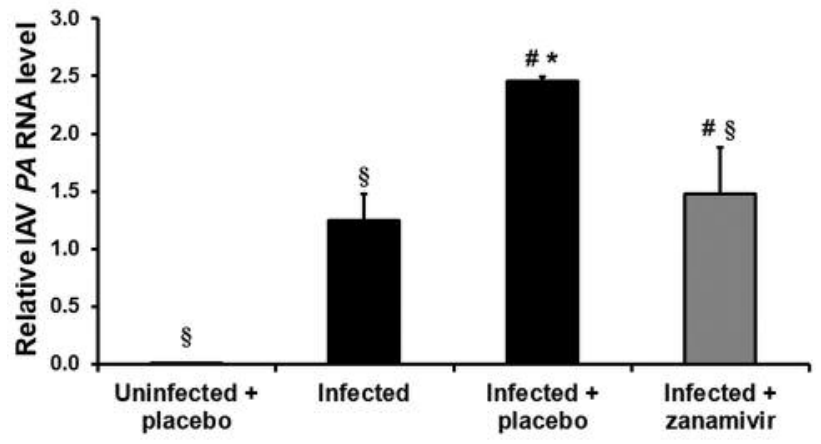

Figure 1. Relative RNA levels of viral polymerase acidic (PA) protein in lungs of treatment and control groups of mice on day 3 after influenza A virus (IAV) infection. Data are shown as mean \pm SEM. Significantly different at $p<0.05 \mathrm{vs} .{ }^{\#}$ uninfected placebo-treated mice $(n=3)$, *infected mice without any treatment $(n=3)$, \$infected placebotreated mice $(n=3)$. Infected + zanamivir, $n=4$.

weight, respectively. However, zanamivir-treated mice had lost only $7 \%$ and $9 \%$ of their initial body mass by days 2 and 3. The weight loss in the zanamivir-treated group was significantly smaller than that of both the infected untreated and infected placebo-treated mice on days 2 and $3(p<0.05)$. 
Table III. The lung histopathology in experimental mouse groups on day 3 after influenza A virus infection.

\begin{tabular}{|c|c|c|c|c|}
\hline & \multicolumn{4}{|c|}{ Mouse group } \\
\hline & Group A, $\mathrm{n}=3$ & Group B, $n=3$ & Group $\mathrm{C}, \mathrm{n}=3$ & Group $\mathrm{D}, \mathrm{n}=4$ \\
\hline Parameter & Uninfected + placebo & Infected & Infected + placebo & Infected + zanamivir \\
\hline Alveolar hemorrhage & $0.33 \pm 0.33$ & $0.33 \pm 0.33$ & $0.33 \pm 0.33$ & 0 \\
\hline Hyperemia & $0.67 \pm 0.33 * \S$ & $0^{\#}$ & $0^{\#}$ & $0 \#$ \\
\hline Focal leukocytic infiltration & $0^{*}$ & $1.33 \pm 0.33^{\# \S}$ & $0^{*}$ & $0^{*}$ \\
\hline Diffuse leucocytic infiltration & 0 & 0 & $0.67 \pm 0.67$ & 0 \\
\hline Perivascular lymphocytic infiltration & $0^{*}$ & $1.00 \pm 0.00^{\# \S}$ & $0^{*}$ & $0.50 \pm 0.29$ \\
\hline Peribronchial lymphocytic infiltration & $0^{*}$ & $1.00 \pm 0.00^{\#}$ & $0.67 \pm 0.33$ & $0.25 \pm 0.25^{*}$ \\
\hline Bronchiolitis & 0 & $0.67 \pm 0.33$ & $0.33 \pm 0.33$ & $0.50 \pm 0.29$ \\
\hline Histological score ${ }^{a}$ & $1.00 \pm 0.58 *$ & $4.33 \pm 0.88^{\# \S}$ & $2.00 \pm 0.00 *$ & $1.25 \pm 0.75^{*}$ \\
\hline
\end{tabular}

aMean cumulative histological score. Significantly different at $p<0.05 v s$. \#group A, *group B, and \$group C.

Pulmonary virus levels. Viral PA RNA was not detected in uninfected control mice whilst increased viral loads were present in the lungs of all groups of IAV-infected mice as quantified by multiplex one-step RT-qPCR assay at day 3 p.i. The infected group treated with lactose had significantly $(p<0.05)$ higher IAV PA RNA level compared with the group that was infected without treatment. Of note, at $2 \mathrm{mg} / \mathrm{kg}$ zanamivir, IAV PA RNA levels were 1.7-fold less than in the infected plus placebo-treated mice $(p=0.05)$. The relative viral PA RNA levels in experimental mouse groups on day 3 after IAV infection are shown in Figure 1.

Mouse lung histopathology. As shown in Table III, the IAV challenge in mice induced acute pulmonary inflammation marked by alveolar hemorrhage, focal leukocytic infiltration, perivascular and peribronchial lymphocytic infiltration and bronchiolitis on day 3 p.i., whereas these histopathological changes, except for alveolar hemorrhage and hyperemia, were absent from the uninfected mice receiving placebo. In the lungs of IAV-infected and placebo-treated mice, alveolar hemorrhage, diffuse leukocytic infiltration, peribronchial lymphocytic infiltration and bronchiolitis were observed. Treatment of the IAV-infected mice with zanamivir resulted in significantly less intense focal leukocytic infiltration and peribronchial lymphocytic infiltration compared with IAVinfected untreated mouse group. We did not observe alveolar macrophages, damage to alveolar/bronchial epithelium, capillary thromboses, pleuritis, collapse, fibrosis, edema or hyaline membranes in lungs of experimental mice. The mean cumulative histological score in the zanamivir-treated group was significantly smaller than that for the IAV-infected untreated mouse group. The photographs of experimental group mice lungs are shown in Figure 2.

Nitrite concentration in BAL fluid. Enhanced NO synthesis in the lungs of both infected control mice at day 3 p.i. was observed, since the measured nitrite concentration in BAL fluids of these mice was $\sim 1.6$-fold higher than in the uninfected control animals (Figure 3). On the other hand, zanamivir-treated IAV-infected mice had lower nitrite concentration in their BAL fluids by $56-58 \%$ than the infected controls ( $p=0.023 v s$. infected untreated, and $p=0.017 v s$. infected placebo-treated).

Relative iNOS mRNA level in mice lungs. As presented in Figure 4, IAV infection caused significant up-regulation of iNOS gene resulting in 2 .6-fold higher mRNA levels compared to uninfected placebo-treated mice $(p<0.05)$. However, zanamivir therapy of IAV-infected mice downregulated iNOS gene expression by $\sim 1.5$-fold versus the infected controls. The inhibitory effect of zanamivir on the production of iNOS mRNA was significant in comparison with untreated infected mice $(p=0.006)$, as well as with infected mice receiving placebo $(p=0.004)$.

\section{Discussion}

The present study demonstrated that a relatively low dose of zanamivir ( $2 \mathrm{mg} / \mathrm{kg} /$ day) was able to limit mouse weight loss and pulmonary viral load, inflammation, NO and iNOS mRNA levels in IAV-infected mice. These findings are specifically important in the context of the third day. Day 3 is the day on which severe IAV infection in humans often progresses to primary viral pneumonia and acute respiratory distress syndrome (11). In fact, IFN- $\gamma$ and TNF- $\alpha$, i.e. the cytokines responsible for induction of iNOS, are markedly produced during the first two days of human IAV infection (12). Likewise, levels of these cytokines begin to rise on day 3 after IAV challenge in mice, leading to increased synthesis of NO, with consequent lung injury $(5,13)$. In this context, our data showed that such augmented NO generation and pulmonary pathology are considerably suppressed by early zanamivir treatment initiated at $3 \mathrm{~h} \mathrm{p.i.} \mathrm{It} \mathrm{is} \mathrm{reasonable} \mathrm{to} \mathrm{relate} \mathrm{these}$ 




Figure 2. Histological lung sections of BALB/c mice stained with hematoxylin and eosin. Arrows indicate: (i) alveolar hemorrhage, (ii) focal leukocytic infiltration, (iii) perivascular lymphocytic infiltration, (iv) peribronchial lymphocytic infiltration, (v) bronchiolitis.

effects to the inhibition of IAV replication by zanamivir causing the decrease of viral load and inflammation. Indeed, zanamivir treatment led to reduced IAV PA RNA level and NO concentration, together with a reduction of iNOS RNA level compared with IAV-infected and placebo-treated mouse groups. However, the IAV PA RNA level was higher in infected lactose treated mice compared with untreated influenza-infected mice in our study, which could be interpreted to mean that the intranasal treatments themselves may facilitate viral replication (14). Surprisingly, although the zanamivir dose of $2 \mathrm{mg} / \mathrm{kg}$ showed no decrease in IAV PA RNA level, the inhibitory effect on NO production together with diminished iNOS RNA level 


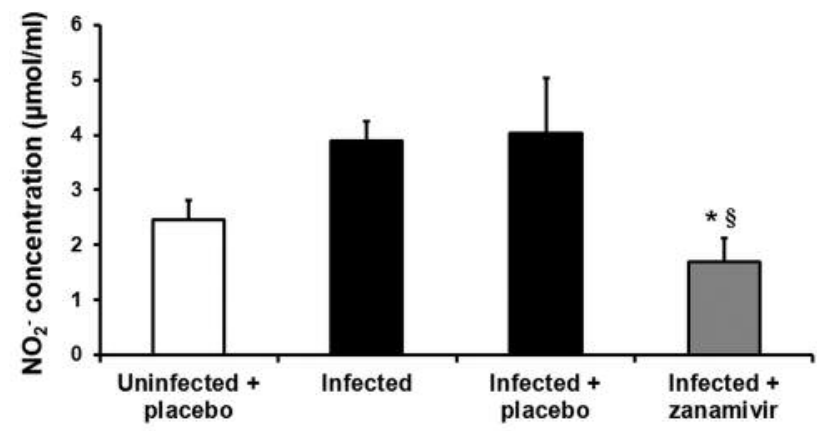

Figure 3. Nitrite $\left(\mathrm{NO}_{2}^{-}\right)$concentration in mice bronchoalveolar lavage fluids. Data are shown as mean \pm SEM. Significantly different at $p<0.05 \mathrm{vs}$. *infected mice without any treatment $(n=3)$, and \$infected placebo-treated mice $(n=3)$. Uninfected + placebo, $n=3$; infected + zanamivir, $n=4$.

was significant compared with influenza infected untreated mice. Thus, it is possible that there is a direct inhibitory effect of zanamivir on NO production in IFN- $\gamma$-activated macrophages, as was previously demonstrated by experiments in vitro (9). It is also possible that the effects of zanamivir on viral entry and cell lysis reduce net inflammation simply by limiting tissue necrosis and the effect of necrosis on generating non-specific inflammation that includes $\mathrm{NO}$ and macrophage activation.

Accordingly, we conclude that zanamivir has therapeutic potential to reduce pulmonary damage in IAV-infected mice through a mechanism involving suppression of NO synthesis. These findings are relevant for the development of new treatment approaches to influenza infection and may also support a beneficial role of zanamivir in early initiation of influenza therapy for preventing severe pneumonia.

\section{Acknowledgements}

This study was supported by the Glennan Center for Geriatrics and Gerontology, EVMS (U.S.A.) and the Faculty of Medicine, Vilnius University (Lithuania). The Authors thank Bradley Bender, University of Florida, for providing the virus.

\section{References}

1 World Health Organization: Influenza (seasonal) fact sheet 2018. World Health Organization, 2018. http://www.who.int/ mediacentre/factsheets/fs211/en/

2 Short KR, Kroeze EJ., Fouchier RA and Kuiken T: Pathogenesis of influenza-induced acute respiratory distress syndrome. Lancet Infect Dis 14: 57-69, 2014.

3 Zaki MH, Akuta T and Akaike T: Nitric oxide-induced nitrative stress involved in microbial pathogenesis. J Pharmacol Sci 98: 117-129, 2005.

4 Akaike T, Noguchi Y, Ijiri S, Setoguchi K, Suga M, Zheng YM, Dietzschold B and Maeda H: Pathogenesis of influenza virusinduced pneumonia: involvement of both nitric oxide and oxygen radicals. Proc Natl Acad Sci USA 93: 2448-2453, 1996.

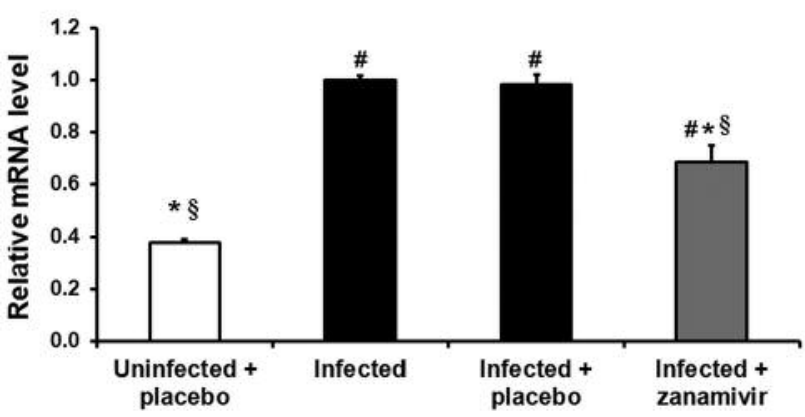

Figure 4. Relative levels of inducible nitric oxide synthase (iNOS) mRNA in mouse lung tissues. Data are shown as mean \pm SEM. Significantly different at $p<0.05 \mathrm{vs} .{ }^{*}$ uninfected placebo-treated mice $(n=3)$, *infected mice without any treatment $(n=3)$, and sinfected placebo-treated mice $(n=3)$. Infected + zanamivir, $n=4$.

5 Perrone LA, Belser JA, Wadford DA, Katz JM and Tumpey TM: Inducible nitric oxide contributes to viral pathogenesis following highly pathogenic influenza virus infection in mice. J Infect Dis 207: 1576-1584, 2013.

6 Ryan DM, Ticehurst J, Dempsey MH and Penn CR: Inhibition of influenza virus replication in mice by GG167 (4-guanidino2,4-dideoxy-2,3-dehydro- $\mathrm{N}$-acetylneuraminic acid) is consistent with extracellular activity of viral neuraminidase (sialidase). Antimicrob Agents Chemother 38: 2270-2275, 1994.

7 Tarbet EB, Hamilton S, Vollmer AH, Luttick A, Ng WC, Pryor M, Hurst BL, Crawford S, Smee DF and Tucker SP: A zanamivir dimer with prophylactic and enhanced therapeutic activity against influenza viruses. J Antimicrob Chemother 69: 2164-2174, 2014.

8 Heneghan CJ, Onakpoya I, Thompson M, Spencer EA, Jones M and Jefferson T: Zanamivir for influenza in adults and children: systematic review of clinical study reports and summary of regulatory comments. BMJ 348: g2547.1-g2547.16, 2014.

9 Kacergius T, Ambrozaitis A, Deng $\mathrm{Y}$ and Gravenstein S: Neuraminidase inhibitors reduce nitric oxide production in influenza virus-infected and gamma interferon-activated RAW 264.7 macrophages. Pharmacol Rep 58: 924-930, 2006.

10 Livak KJ and Schmittgen TD: Analysis of relative gene expression data using real-time quantitative PCR and the 2(-Delta Delta C(T)) method. Methods 25: 402-408, 2001.

11 Rello J and Pop-Vicas A: Clinical review: Primary influenza viral pneumonia. Crit Care 13: 235, 2009.

12 Kaiser L, Fritz RS, Straus SE, Gubareva L and Hayden FG: Symptom pathogenesis during acute influenza: interleukin- 6 and other cytokine responses. J Med Virol 64: 262-268, 2001.

13 Suliman HB, Ryan LK, Bishop L and Folz RJ: Prevention of influenza-induced lung injury in mice overexpressing extracellular superoxide dismutase. Am J Physiol Lung Cell Mol Physiol 280: 69-78, 2001.

14 Smee DF, Itzstein M, Bhatt B, Tarbet EB: Exacerbation of influenza virus infections in mice by intranasal treatments and implications for evaluation of antiviral drugs. Antimicrob Agents Chemother 56(12): 6328-6333, 2012.

Received February 11, 2018

Revised March 11, 2018

Accepted March 14, 2018 